\title{
Criterios de documentación no invasivos para el estudio y difusión del arte rupestre
}

\section{Manuel Bea ${ }^{a}$ y Jorge Angás}

aÁrea de Prehistoria, Dpt. Ciencias de la Antigüedad, Universidad de Zaragoza. C/ Corona de Aragón 42, 50009, Zaragoza. Miembro del Comité Nacional de ICOMOS. manubea@unizar.es, ${ }^{b}$ ETSI Topografía, Geodesia y Cartografía, Universidad Politécnica de Madrid, Campus Sur UPM, Autovía de Valencia km. 7, 28031, Madrid. Miembro del Comité Nacional de ICOMOS. j.angas@usal.es.

\begin{abstract}
Resumen
Todo estudio relacionado con el Patrimonio, sea cual sea la naturaleza de éste, encuentra en la documentación una de las bases esenciales para su correcto desarrollo. Esa documentación, que puede encontrar diferentes métodos y técnicas en función del objetivo (investigación, administración, conservación, didáctica, difusión...), ha de ser lo más exhaustiva posible pero siempre atendiendo a parámetros y criterios de conservación. El arte rupestre es un bien patrimonial excepcional y singular por múltiples aspectos y presenta dificultades en su documentación que determinan una falta de normativa homogénea y globalmente aceptada. En este trabajo abordamos la propuesta de un sistema de documentación integral e integrador aplicado en numerosos ejemplos, tanto paleolíticos como postpaleolíticos, que hemos llevado a cabo desde la investigación pero siempre atendiendo a diversos propósitos teniendo en cuenta, como un elemento básico, la conservación y protección de los conjuntos rupestres.
\end{abstract}

Palabras clave: arte rupestre, documentación, calcos digitales, fotogrametría, escáner láser, investigación, conservación, difusión.

\begin{abstract}
Every study related to Heritage, whatever its nature, finds an essential bases for its development on the documentation. This field, with different methods and techniques depending on the goal (research, administration, preservation, didactics, diffusion...), must be exhaustive and also absolutely thorough with preservation criteria. Rock Art is an exceptional and singular type of heritage attending to different aspects. The difficulty on its documentation points out a lack an accepted global and homogeneous regulation. In this paper we focus on a proposal of a integral documentation system applied to a large number of cases of study, both Palaeolithic and post-Palaeolithic, that we have carried out from research projects but attending to different purposes taking into account, as a key guideline, the preservation and protection of the sites.
\end{abstract}

Keywords: rock art, documentation, digital tracing, photogrammetry, laser scanner, research, preservation, dissemination. 


\section{Introducción}

La documentación es el primer eslabón en la cadena de estudio de cualquier bien patrimonial, resultando esencial en aquellos campos que encuentran en el componente gráfico el principal valor de aproximación a su realidad. En esta línea, el arte rupestre quizá sea uno de los bienes más paradigmáticos. Se trata de un contexto material excepcional, íntimamente vinculado al ser humano, atemporal y pangeográfico, que permite acercarnos a aquellas parcelas más etéreas de las sociedades pretéritas: simbolismo, sentimiento y pensamiento. A pesar de su amplia distribución mundial y de su relativa abundancia, se trata de un elemento frágil y, en muchos casos y contextos, de compleja visualización.

En el último decenio se han generalizado los métodos y técnicas no invasivos para la documentación del patrimonio en general (Angás, 2019) y para el arte rupestre en particular. Este campo es uno de los más destacados dada la singularidad del objeto de estudio. En estas páginas presentamos una propuesta de protocolo de documentación gráfico no invasivo aplicado al arte rupestre cuya viabilidad y buenos resultados hemos podido contrastar mediante su aplicación en numerosos abrigos y cuevas con arte rupestre. En todo caso, el registro en general, que debe tener como objetivo la reproducción objetiva de la realidad documentada, así como los sistemas de captación de datos y métodos aplicados debe atender a una diversidad de fines: administración, investigación, conservación, difusión... de manera que sus utilidades se deben refrendar atendiendo a diferentes niveles con el objetivo común de conocer fielmente el elemento representado, con la misión de asegurar y promover la conservación, protección, uso y desarrollo de este bien patrimonial (Fig. 1).

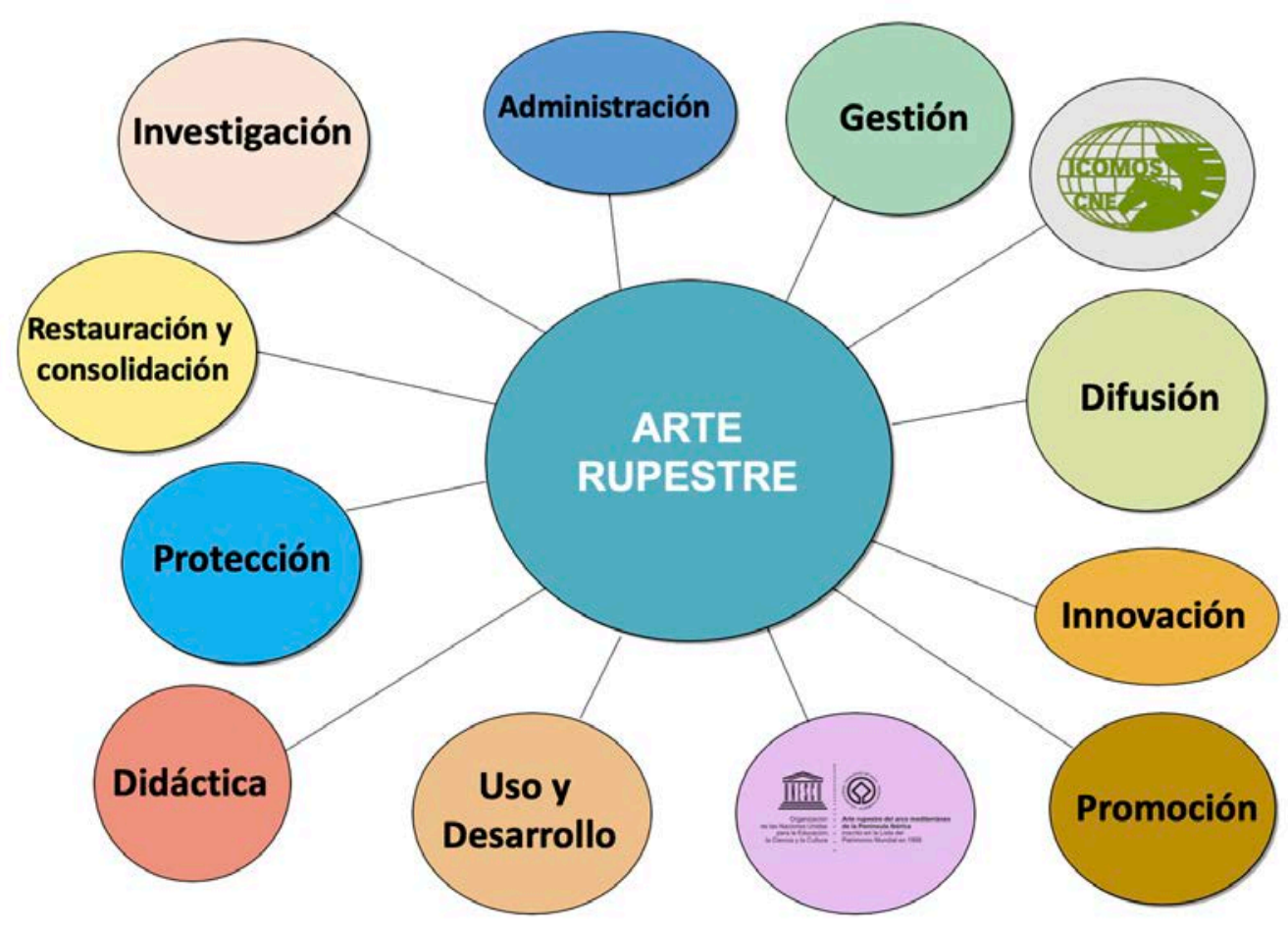

Conocer fielmente el motivo representado

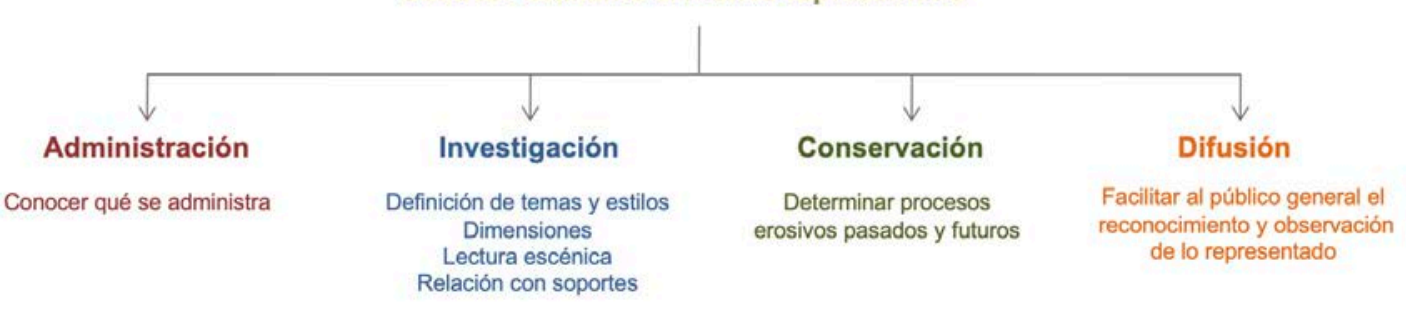

Fig. 1 Esquema de los fines básicos de la documentación según los diferentes objetivos 
Atendiendo al arte rupestre, la aplicación de determinadas tecnologías ha supuesto una verdadera revolución con la que se ha conseguido aumentar la calidad de los resultados, reducir tiempos de trabajo, mejorar la conservación de lo documentado, ampliar posibilidades de análisis, integrar resultados y acceso a metadatos o diversificar canales de difusión... Con todo, a la tradicional fórmula de I+D+i se debería añadir una nueva "d", de manera que la difusión aparezca como elemento consustancial en todo estudio patrimonial.

Siguendo las premisas de no afección sobre el objeto documentado y de la multiplicidad de usos de los resultados, el Sistema de Documentación Integral propuesto conforma un protocolo de actuación no invasivo para el arte rupestre en el que se contempla la fotografía digital, elaboración de calcos digitales y documentación geométrica mediante diferentes sistemas y herramientas (láser escáner, escáner de luz blanca estructurada y fotogrametría).

\section{Metodologías}

El Sistema de Documentación Integral (SDI) planteado y desarrollado durante los últimos años integra diferentes métodos y técnicas de obtención de datos no invasivos, esencialmente centrados en imágenes bidimensionales (fotografía y calcos digitales) y documentación geométrica para la obtención de modelos tridimensionales, mediante el uso de láser escáner, escáner de luz blanca estructurada y/o fotogrametría, según las necesidades intrínsecas de cada conjunto (Angás y Bea, 2019a). Los resultados obtenidos permiten a su vez hacer un uso combinado de los mismos para generar nuevos elementos con información gráfica susceptibles de aportar información complementaria (visual o métrica) en función los fines planteados así como de las plataformas o medios empleados: combinación de calcos sobre modelos tridimensionales, archivos para consulta y visualización en navegadores (con la especificación estándar WebGL) que permiten implementar, incluso, precisas herramientas de medición sobre los modelos o aplicación de filtros (Angás y Collado, 2018).

Atendiendo al primer bloque de métodos de documentación no invasiva referido (imágenes bidimensionales), el procedimiento planteado se basa en la elaboración de calcos prescindiendo por completo de cualquier tipo de intervención directa sobre el motivo rupestre que pueda implicar el contacto con el mismo y su humectación. La esencia del método descansa en prácticas previas de calcos indirectos llevados a cabo sobre fotografías impresas o imágenes proyectadas, sirviéndonos de las mejoras ofrecidas por el avance en materia de fotografía digital y su tratamiento informático.

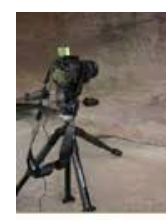

Toma fotográfica de cada motivo

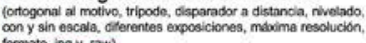
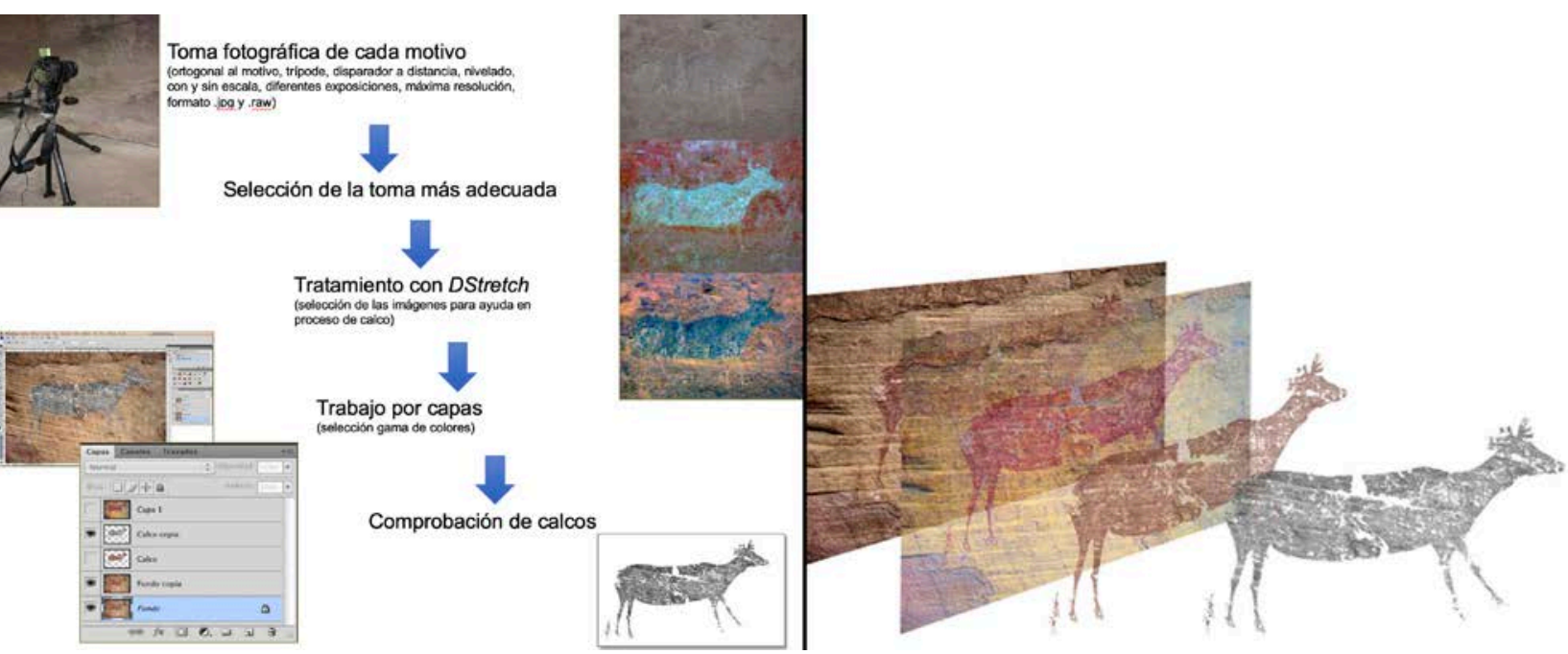

Fig. 2 Proceso de confección del calco digital sobre pinturas levantinas

En términos generales, el calco se puede definir como un elemento/medio de trabajo para tratar de responder a problemáticas tanto de investigación como de análisis de diferentes campos, pero encuentra igualmente aplicación en materia de difusión. Se trata de un registro gráfico llevado a cabo en un momento concreto que mejora la visibilidad del objeto de estudio. Es, por tanto, un producto que aporta información sustancial para el correcto desarrollo del estudio y 
diagnosis. Naturalmente, la información con la que se debe contar ha de ser lo más precisa posible respecto de la realidad, es decir, objetiva. Esa pretendida objetividad no se ha alcanzado en lo referido a la realización, plasmación y presentación de calcos rupestres empleando técnicas de calco directo o indirecto analógico. Esas diferencias, si bien no siempre tan marcadas, son las que han determinado que surja, en determinados sectores, voces críticas acerca de la necesidad de realizar y emplear calcos. Sin embargo, a partir de nuestra experiencia, el calco se posiciona como herramienta imprescindible en el estudio del arte rupestre con múltiples aplicaciones tangenciales. Ni la fotografía ni el tratamiento digital de la imagen pueden sustituir (en todo caso complementan) las posibilidades de identificación gráfica y de presentación de resultados que aporta un calco. El uso de determinadas tecnologías y la observación de un protocolo bien estructurado nos ha permitido, en los últimos años, llevar a cabo calcos con mayor grado de objetividad mediante la aplicación de otras herramientas digitales, ofreciendo grandes resultados con una reducción muy considerable de los costes (en tiempo, esfuerzo y económico) y nula afección para la conservación de los motivos rupestres (Bea, 2012; Domingo, 2014; 2019) (Fig. 2).

Sus posibilidades de aplicación contemplan también el uso combinado de otros sistemas de documentación, como fotogrametría y láser escáner, ampliando la profundidad de análisis en materia de conservación y difusión (Fig. 3). La validez del método (tanto para pintura como para grabado) no sólo se constata como medio de estudio (esencialmente orientado a la investigación) sino también, y no menos importante, como elemento para la difusión de este bien patrimonial en diferentes plataformas y para diferentes públicos contribuyendo a la conservación, protección y puesta en valor de este singular patrimonio cultural.
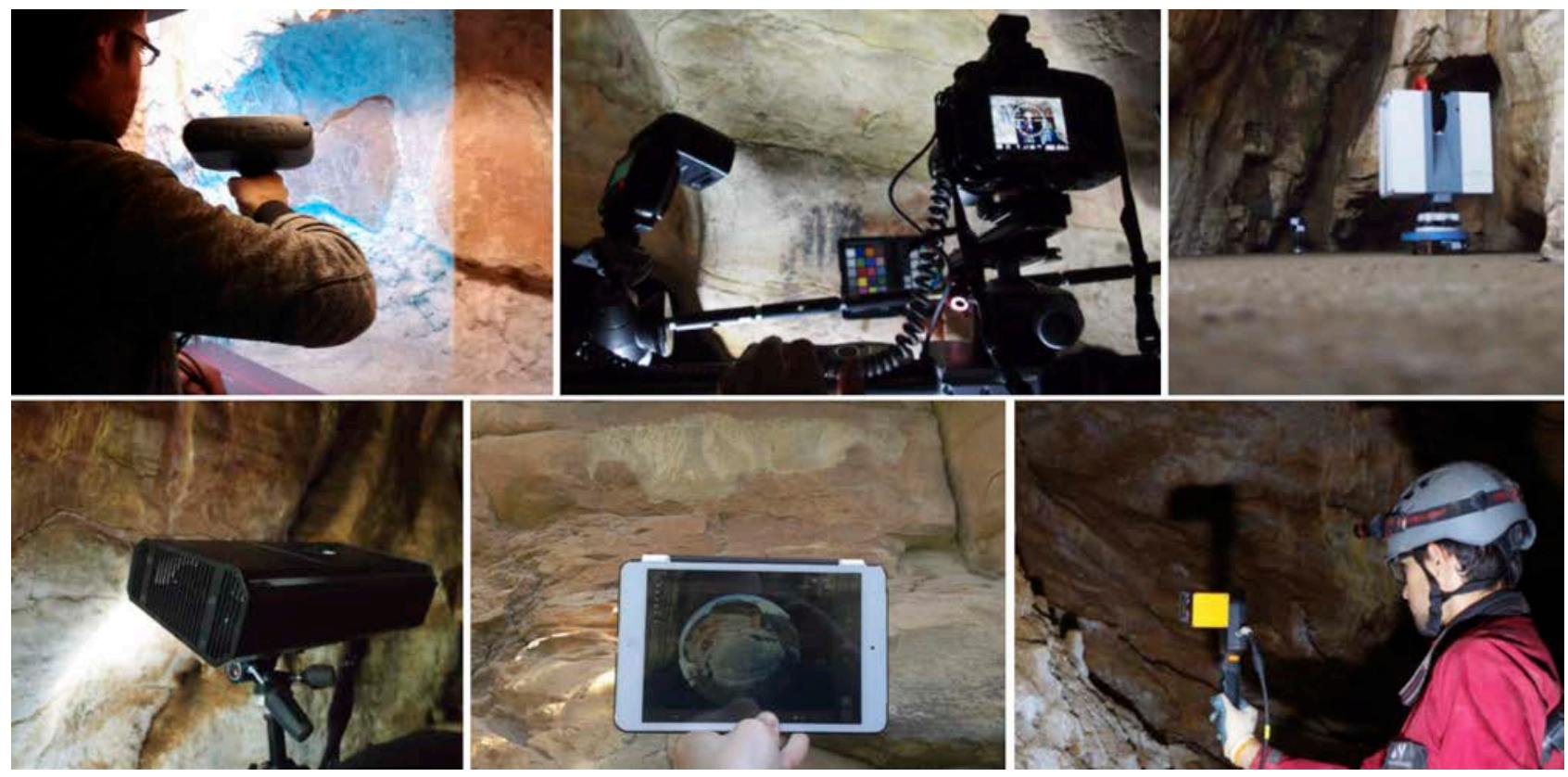

Fig. 3 Diferentes sistemas de documentación tridimensional aplicados en conjuntos rupestres

Junto a lo anterior, la documentación geométrica aparece como un campo de obtención de datos de primera magnitud, empleándose para ello diversos métodos y técnicas adaptadas a las particularidades del bien patrimonial. El arte rupestre, nuevamente, aparece como un elemento de la cultura material de carácter singular ya que, para su utilidad científica, deberá contarse con la máxima calidad gráfica y métrica al tiempo que permita una fácil gestión y se establezcan estándares de clasificación de datos que permitan su trazabilidad futura. Asimismo, cada conjunto con arte rupestre (cueva, abrigo, bloque exento) presenta unas particularidades propias que determinarán el proceso de captación de información en campo, algo que también estará en función de la finalidad de la propia documentación (Fig. 1). Atendiendo a estos particulares las herramientas empleados se sintetizan en tres categorías: topografía de apoyo; escáneres (láser escáner y de luz blanca estructurada); y fotogrametría terrestre.

La información generada a partir de estos sistemas permite la confección de modelos tridimensionales de gran precisión y resolución a partir de los cuales se puede acceder a información acerca de la coservación del conjunto (y su 
evolución diacrónica con documentaciones puntuales a lo largo del tiempo) (Bea y Angás, 2014), plantear un amplio abanico de posibilidades relacionadas con la difusión (recorridos virtuales, restituciones, réplicas...) (Angás y Bea, 2014; 2015; 2019a; Bea y Angás, 2016), gestión (bases de datos relacionales...) (Angás, 2019) y, naturalmente, con la investigación. Todo ello aporta un concepto diferente en el registro, análisis y valorización que consigue un registro diverso y multifuncional a través de la estandarización de procesos (control de calidad y comprobación), integración de aplicaciones gráficas y métricas, gestión de datos tridimensionales, difusión de resultados, etc.

\section{Resultados}

Como se puede traslucir de las páginas precedentes, los resultados obtenidos mediante un SDI como el propuesto permiten abordar un amplio campo de aplicaciones para los conjuntos rupestres atendiendo a campos y funciones diversificados, todos ellos igualmente importantes en la consideración global y valorización de un bien excepcional como el que nos ocupa. Quizá un ejemplo aglutinante de las técnicas no invasivas referidas en el anterior apartado pueda referirse la documentación y restitución de los conjuntos rupestres destruidos de la cuenca del Matarraña (Bea y Angás, 2017; Angás y Bea, 2019b), en el que se articulan valores no sólo relacionados con la investigación, administración, gestión o conservación si no también con otros conceptos inherentes al arte rupestre como puedan ser su propia valorización a través de una difusión de calidad. Mediante ésta se pretende, igualmente, despertar la concienciación pública acerca de la importancia del arte rupestre y de la necesidad de protegerlo, al tiempo que puede actuar como un elemento complementario dentro de una oferta cultural más amplia que incentive el desarrollo socioeconómico del territorio.

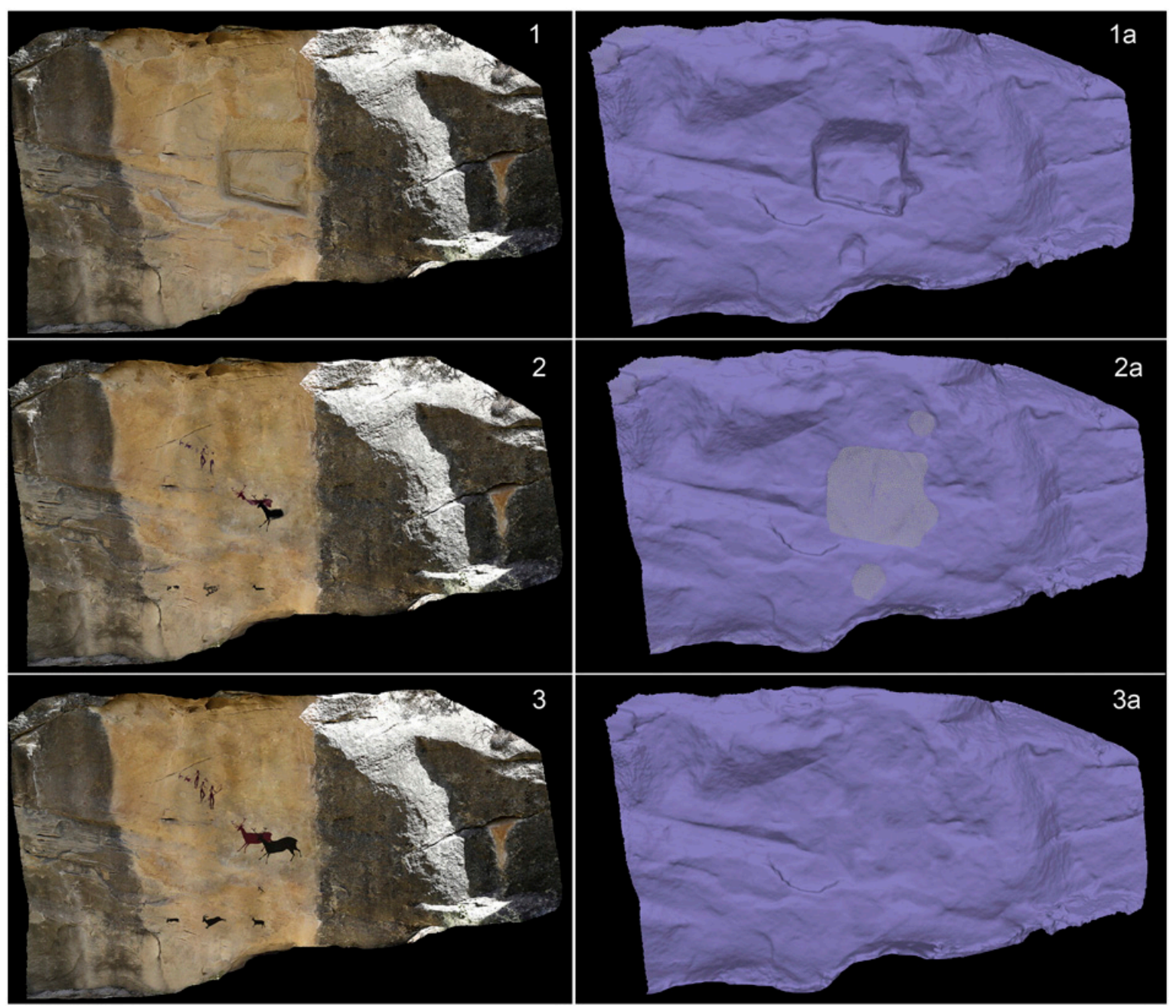

Fig. 4 Reconstrucción hipotética del abrigo de Els Gascons (según Bea y Angás, 2017, p. 165) 
Así pues, partimos de la destrucción muy temprana de distintos conjuntos rupestres en la zona del Matarraña (Teruel), en diferentes momentos y por diferentes causas (Martínez-Bea, 2005), motivo por el cual el proyecto se centró en la recuperación virtual de los conjuntos rupestres, tratando de llevar a cabo una propuesta de evolución diacrónica de los restos todavía conservados. Todo ello ahondando en la necesidad de que la investigación permitiera llevar a cabo una mejora real en la comprensión y conocimiento general del arte rupestre, así como de la concienciación social de su valor y fragilidad (Domingo y Bea, 2016).

Para ello se documentaron los abrigos rupestres de Roca dels Moros, Els Gascons, Caídas del Salbime y Els Secans así como los bloques arrancados del primero de los conjuntos (conservados en el Museu d'Arqueologia de Catalunya) empleando para ello fotografía digital y diferentes métodos de documentación geométrica: láser escáner, escáner de luz blanca estructurada y fotogrametría, además de llevar a cabo fotografías equirrectangulares que permitirían la realización de diferentes presentaciones. Los datos obtenidos (mediante sistemas no invasivos) y los productos generados a partir de éstos se combinaron permitiendo la restitución digital de elementos perdidos, destruidos o removidos (Fig. 3).

La presentación de resultados se realizó mediante a generación de una página web y de una aplicación para dispositivos móviles ("Matarranya Rock Art"), criterio que resolvió de forma eficiente algunas de las problemáticas que se plantean en todo proyecto de estudio y documentación patrimonial como el uso amplio y combinación de los datos obtenidos y su presentación al público general, ofreciendo nuevas posibilidades de visualización de un bien patrimonial desaparecido, aportando un equilibrio entre información científica y difusión interactiva.

\section{Conclusiones}

El arte rupestre es un bien patrimonial singular y excepcional de carácter atemporal y pangeográfico, es decir, global y consustancial al ser humano y que, por tanto, le ha valido un reconocimiento internacional que se traduce, en muchos casos, en su integración dentro del listado Patrimonio Mundial de la UNESCO (Sanz, 2008; 2012). Sin embargo, hay muchos factores que determinan su gran fragilidad (antigüedad, agentes naturales, climatología, antrópicos...).

Hasta no hace mucho, prácticamente los únicos medios disponibles para la documentación del arte rupestre implicaban el contacto directo con el motivo y el soporte, afectando de forma meridiana a la conservación o corto-medio plazo de los conjuntos. La implementación de nuevas técnicas y métodos (todos de ellos procedentes de otros campos de estudio y análisis que han debido ser adaptados a las necesidades de la investigación arqueológica) nos permiten obtener mejores resultados, con menor coste global y sin afección alguna sobre los paneles decorados. El SDI referido en estas líneas se entiende sólo como una propuesta personal desarrollada y adaptada a diferentes realidades a lo largo de los últimos años, encontrando procedimientos afines en diferentes contextos institucionales y geográficos, entendidos como respuestas similares ante problemáticas comunes (Defrasne, 2014; Cortón et al., 2015; Domingo, 2014; 2019; Cabrelles y Lerma, 2015; Lerma et al., 2013).

La constatación de una degradación (por otra parte consustancial) en crecimiento exponencial de muchos conjuntos rupestres nos debe hacer reflexionar en campos muy diversos. La obligación de proteger y conservar debería tratar, al menos y en primera instancia, de relantizar el proceso de deterioro y, en segundo lugar, contar con la mejor documentación no invasiva posible que nos permita contar con una especie de repositorio o reservorio patrimonial (siquiera digital) para generaciones futuras. En todo caso, en el actual estado de desarrollo y aplicabilidad tecnológica ya no se justifica el uso de otros sistemas de documentación en los que se interviene de forma directa en el bien.

\section{Agradecimientos}

El presente trabajo se enmarca en la línea de estudio y documentación integral de arte rupestre del Proyecto MINECO HAR2017-85023-P y del Grupo de Investigación Primeros Pobladores y Patrimonio Arqueológico (H1417R) (Gobierno de Aragón y Fondo Social Europeo). 


\section{Referencias}

Angás, J. (2019). Documentación geométrica del patrimonio cultural. Análisis de las técnicas, ensayos y nuevas perspectivas. Caesaraugusta, 86. Zaragoza: Institución Fernando el Católico.

Angás, J., y Bea, M. (2014). Propuesta conceptual y metodológica en la documentación gráfica y geométrica del arte rupestre: Proyecto ARAM (Arte Rupestre y Accesibilidad Multimedia). En M. A. Medina-Alcaide, A. J. Romero, R. Ruiz-Márquez y J. L. Sanchidrián (Coords.), Sobre rocas y huesos: las sociedades prehistóricas y sus manifestaciones plásticas (pp. 348-365). Córdoba: Fundación Cueva de Nerja y Universidad de Córdoba.

Angás, J., y Bea, M. (2015). Propuesta metodológica para la accesibilidad multimedia del arte rupestre prehistórico. En A. Domínguez, J. García, J. y P. Lavado (Eds.), II Congreso Internacional de Educación y Accesibilidad (pp. 925-934). Huesca: Máster Propio en Museos: Educación y Comunicación. Universidad de Zaragoza.

Angás, J., y Bea, M. (2019a). Abrigos rupestres: protecto ARAM. En J. Angás (Ed.), Documentación geométrica del patrimonio cultural. Análisis de las técnicas, ensayos y nuevas perspectivas. Caesaraugusta, 86 (pp. 88-95). Zaragoza: Institución Fernando el Católico.

Angás, J., y Bea, M. (2019b). Arte rupestre: una propuesta de restauración digital del arte rupestre destruido de los abrigos de Roca dels Moros y Els Gascons (Cretas, Teruel). En J. Angás (Ed.), Documentación geométrica del patrimonio cultural. Análisis de las técnicas, ensayos y nuevas perspectivas. Caesaraugusta, 86 (pp. 146-154). Zaragoza: Institución Fernando el Católico.

Angás, J., y Collado, H. (2018). Metodología de documentación y análisis geométrico para el proyecto HANDPAS mediante microfotogrametría y escáner tridimensional de luz blanca estructurada. En H. Collado (Coord.), HANDPAS: Manos del Pasado. Catálogo de representaciones de manos en el arte rupestre paleolítico de la península Ibérica (pp. 17-28). Mérida: Junto de Extremadura.

Angás, J., Sabah, A., Bea, M., Farjas, M., Uribe, P., Lanau, P., y Otero, X. (2019). Rock Art recording in Khatm al Melaha (United Arab Emirates): multirange data scanning and web mapping technologies. The International Archives of the Photogrammetry, Remote Sensing and Spatial Information Sciences, XLII-2/W15, 85-92. https://doi.org/10.5194/isprs-archives-XLII-2-W15-852019.

Bea, M. (2012). Documentando el arte rupestre pictórico en Aragón. En N. Juste, M. A. Hernández, A. Pereta, J. I. Royo y J. Andrés (Dirs.), Jornadas técnicas para la gestión del arte rupestre, Patrimonio Mundial (pp. 54-60). Huesca: Comarca del Somontano de Barbastro.

Bea, M., y Angás, J. (2014). The Conservation of Spanish Levantine Rock-Art in Aragón, Spain, Using 3-D Laser Scanning. En T. Darvill, y A. P. Batarda (Eds.), Open-air rock art conservation and management (pp. 159-166). London: Routledge.

Bea, M., y Angás, J. (2016). Nuevas metodologías de documentación para investigación y difusión del arte rupestre en Aragón. En J. I. Lorenzo y J. M. Rodanés (Eds.), I Congreso de Arqueología y Patrimonio Aragonés (pp. 523-531). Zaragoza: Colegio Oficial de Doctores y Licenciados en Filosofía y Letras y en Ciencias de Aragón, Zaragoza.

Bea, M., y Angás, J. (2017). Geometric documentation and virtual restoration of the rock art removed in Aragón (Spain). Journal of Archaeological Science: Reports, 11, 159-168. https://doi.org/10.1016/j.jasrep.2016.11.025

Cabrelles, M., y Lerma, J. L. (2013). Documentación 3D de abrigos rupestres a partir de láser escáner y de procesos fotogramétricos automatizados. Virtual Archaeology Review, 4(8), 64-68.

Cortón, N., López, A., y Carrera, F. (2015). Combining photogrammetry and photographic enhancement techniques for the recording of megalithic art in north-west Iberia. Digital Applications in Archaeology and Cultural Heritage, 2, 89-101.

Defrasne, C. (2014). Digital image enhancement for recording rupestrian engravings: applications to an Alpine rock shelter. Journal of Archaeological Science, 50, 31-38. https://doi.org/10.1016/j.jas.2014.06.010

Domingo, I. (2014). Rock art recording methods: from traditional to digital. En C. Smith (Ed.), Encyclopedia of Global Archaeology 6351-6357. New York: Springer.

Domingo, I. (2019). Arte rupestre y tecnologías digitales: una revisión actualizada. En G. García y V. Barciela (Coords.), Sociedades prehistóricas y manifestaciones artísticas. Imágenes, nuevas propuestas e interpretaciones (pp. 201-208). Alicante: Instituto Universitario de Investigación en Arqueología y Patrimonio Histórico. 
Domingo, I., y Bea, M. (2016). From Science to Heritage: new challenges for World Heritage rock art sites in Mediterranean Spain in the Twenty-First century. En L. M. Brady y P. S. C. Taçon (Eds.), Relating to Rock Art in the Contemporary World: navigating symbolism, meaning and significance (pp. 213-244). Boulder: University Press of Colorado.

Lerma, J. L., Cabrelles, M., Navarro, S., y Seguí, A. E. (2013). Modelo fotorrealístico 3D a partir de procesos fotogramétricos: láser escáner versus imagen digital. Cuadernos de Arte Rupestre, 6, 85-90.

Martínez-Bea, M. (2005). Breve aproximación historiográfica a los estudios pioneros sobre arte rupestre en el Bajo Aragón y Maestrazgo turolense. Salduie, 5, 57-63.

Sanz, N. (2008). Introducción. En N. Sanz (Ed.), Arte rupestre en el Caribe. Hacia una nominación transnacional seriada a la Lista del Patrimonio Mundial de la UNESCO (pp. 80-113). Paris: World Heritage Centre, UNESCO.

Sanz, N. (2012). Rock Art and the UNESCO World Heritage List. En J. McDonald y P. Veth (Eds.), A companion to rock art (pp. 491-514). Chicester: Wiley-Blackwell. 